\title{
Basic science for the clinical gastroenterologist: A review of the recent literature on the small bowel (part 2)
}

\author{
F. Urrutia, MD, R.N. Fedorak, MD, FRCPC, S. Churnkatanakul, MD, \\ M. KEELAN. MSC, A.B.R. THOMSON, MD, PHD, FRCPC, FACP
}

$\mathrm{F}$ OR SMALL BOWEL BASIC SCIENCE, AS in all parts of medicine, there has been an explosion of information. This is the second of a two-part series in which the scientific basis upon which clinical gastroenterology practice is based is considered and toward which clinical practice is moving

\section{ETHANOL}

Chronic ingestion of ethanol has a minimal impact on the intestinal absorption of calcium, when assessed in vivo. but renders the brush border membrane less permeable to calcium and less susceptible to the stimulation by ethanol of calcium flux across this membrane (1). Ethanol and other alcohols inhibit the intestinal transport of folates (2). Chronic ethanol ingestion reduces enterocyte turnover in rat jejunum and ileum and increases lactase activity and the maximum capacity for galactose absorption (3). This effect is possibly due to an increased maturity of the enterocyte population of the villus surface in response to ethanol.

In rats with a jejunal self-filling diverticulum (blind-loop), there is a considerable amount of ethanol oxidized in the gastrointestinal lumen. The resulting high concentrations of acetylaldehyde in the intestinal lumen and the portal blood may contribute to the deleterious effect on the gastrointestinal mucosa and liver (4). Conditions associated with excessive proliferation of intestinal microorganisms may thus result in the production of ethanol and acerylaldehyde.

The acute and chronic administration of ethanol inhibits the intestinal absorption of a variety of nutrients. The addi-

Can J Gastroenterol 1988;2(4):165-76

Key Words: Absorption, Motility, Recent advances, Secretion

Nutrition and Metabolism Research Group, Division of Gastroenterology, Department of Medicine, University of Alberta, Edmonton, Alberta

Correspondence and reprints: Dr A.B.R. Thomson, Nutrition and Metabolism Group, Division of Gastroenterology, Department of Medicine, 519 Robert Newton Research Building, University of Alberta, Edmonton T6G 2C2. Telephone (403) 432-6490

Received for publication March 30, 1988. Accepted April 27, 1988

tion of amiloride, a sodium transport blocker, produces substantial increases in sodium dependent solute uptake in isolated villus cells in the presence of ethanol (5). This suggests that activation or development of amiloride sensitive sodium conductance channels may be an early consequence of intestinal villus cell exposure to ethanol.

Acute and chronic ethanol ingestion alters intestinal transport of nutrients, in part due to alterations in the brush border membrane content of phospholipids (6). There is a stimulatory effect of ethanol on the intestinal absorption of choline with both acute and chronic ethanol administration to rats (7). This enhancement in choline absorption is likely related to ethanol metabolism, since there is a latency in this effect of ethanol and because pyrazol, an inhibitor of ethanol metabolism, prevents the increase in choline absorption. Choline uptake is unaffected by acetaldehyde or acetate. This raises the possibility that the enhancing effect of alcohol on choline absorption is related to stimulation by other products of ethanol metabolism such as changes in ATP. ADP and $\mathrm{NADH}$.

Chronic alcohol ingestion in the rat decreases the jejunal villus cell popula- 
tion, increases the crypt cell population, has no effect on the crypt cell production rate and appears to cause a lengthening of the cell cycle time (8). The metaphase arrest technique has also been used to demonstrate a selective increase in the crypt cell production rate in the rectal mucosa of ethanol fed rats (9). Features of this hyperproliferative state in ethanol fed animals are an increased cell production per crypt and an upward extension of the proliferative compartment comprising the lower half of crypts. Mucosal alcohol dehydrogenase activities are increased in rectal mucosa after prolonged ethanol ingestion and this, or the increased serum gastrin concentrations in alcohol treated animals, may give rise to the rectal hyperproliferation. Further investigations are needed to determine whether these findings are also evident in humans, especially with respect to the increased incidence of rectal cancer found in alcoholics.

Intraluminal ethanol in concentrations normally found in the duodenojejunal lumen after moderate drinking causes morphological lesions and alterations in the mucosal microcirculation. Intraluminal ethanol causes microvascular stasis manifested by vasodilatation, hemoconcentration, increased microvascular arteriovenous shunting of blood through nonnutritive microvessels and enhanced secretory filtration of plasma from the mucosal microvasculature into the gut lumen. These changes were accompanied by an enhanced mucosal microvascular permeability (10). Dual-sized isotope labelled microspheres have demonstrated microvascular stasis manifested by vasodilatation, hemoconcentration, increased microvascular arteriovenous shunting of blood through the nonnutritive microvessels and enhanced secretory filtration of plasma from the mucosal microvasculature into the gut lumen. Using colloidal carbon vascular labelling technique, it has been demonstrated in dogs that $6 \%$ intraluminal ethanol causes marked accumulation of carbon particles in the walls of the capillaries and microvenules of the villus tips, localized between the endothelial cell and the basolamina of the microvessels. The escape of these particles from the lumen probably occurs through cell junctions.
The remarkable similarity of these findings and those following the production of acute inflammation raise the possibility that the known release or production of some of the mediators of acute inflammation may be responsible for the ethanol associated changes in mucosal microvascular permeability (10).

\section{DEVELOPMENT, ONTOGENY AND AGEING}

The small intestine of rats undergoes a concert of morphologic and enzymatic changes during the latter half of the third week after birth. These developmental changes result in a mature intestine functionally optimal for the digestion and absorption of the carbohydrate rich diet of adults. A mong the enzymatic changes, lactase activity decreases and sucrase activity appears and increases. This morphologic and enzymatic differentiation is considered to be initiated by an ontogenic timing mechanism which is mod. ulated by extrinsic, systemic and luminal factors. For example, an ontogenic timing mechanism likely initiates the expression of sucrase activity (11).

The initiation of the post natal ontogenic events in the rat gastrointestinal tract is probably determined by the animal's genetic program, while the terminal phase of intestinal development seems to be influenced more by environmental factors such as nutrients, hormones and other mediators. The morphological and functional development of the intestine has been the subject of a recent review (12).

Maturation of gastrointestinal function during the perinatal period is accompanied by substantial changes in the gastrointestinal surface. These differences include: higher lipid to protein ratios: more fluidity and disorganized structure; the presence of an additional calcium binding site contributing to the higher calcium binding capacity of the brush border membrane of neonates; greater binding of cholera toxin to the mature brush border membrane; and alterations in binding of concanavalin $\mathrm{A}$ as well as other lectins (13).

Small intestinal epithelium is more permeable in the neonatal period than after weaning. 'Closure' or cessation of immunoglobulin absorption occurs at weaning with concomitant modifications of epithelial renewal and function. In humans, which receive their passive immunity exclusively before birth, nonimmunoglobulin macromolecular transfer is also thought to be high during the neonatal period because of the immaturity of the intestinal mucosa. Horseradish peroxidase is transported by way of two functional pathways; a major route involving lysosomal degradation and a minor route allowing transport of the intact protein. There appears to be a rate limiting step in the absorption before the lysosomal system, presumably at the brush border membrane, and development of these two transcellular pathways for nonimmunoglobulin protein absorption is not parallel and does not correlate with gut closure to immunoglobulin transfer (14).

It is generally assumed that breast milk is specifically tailored to the nutritional and developmental needs of the newborn. The differences between colostrum and feeding artificial food formulas have been attributed to one of the trophic factors known to be present in colostrum and human milk. The post natal development changes in small intestinal enzyme activity are complex and multifactorial, including genetic programming, hormones and dietary components. Lactose may have an important function in the stabilization of newborn intestinal disaccharidase enzyme (15), thereby preserving rather than inducing enzyme activity. Lactose may thus have a role in the function of the developing small intestine along with other multiple 'growth factors' present in human milk.

Several factors have been proposed to regulate the physiological events which surround the developmental changes which occur in the small intestine during the post natal period. The factors which have been proposed include glucocorticoids, insulin, thyroxin and epidermal growth factors. High concentrations of epidermal growth factors are found in mouse and rat milk throughout lactation with maximal levels between approximately one week and declining towards weaning. At this time there is a marked increase in endogenous epidermal growth factor produc- 
tion from the neonate salivary glands and intact epidermal growth factor may be absorbed in the small intestine. Epidermal growth factor receptors are present in mouse small intestine with minimal binding at birth. This binding increases steadily throughout post natal life to reach adult values by approximately one month of age (16). These points all raise the possibility that epidermal growth factor is a modulator in the functional development of the maturing gastrointestinal tract. Suckled newborn animals have a greater intestinal growth than animals which are fed water or ar tificial formula; breast-milk fed animals also have heavier intestines which contain more DNA than those given artificial formula (17).

Human transforming growth factor binds to an epithelial cell line with reduction in thymidine incorporation, yet promotion of sucrase activity (18). This suggests that transforming growth factor may inhibit proliferative activity and promote adult development of differentiated function in intestinal epithelial cells.

The epithelial cell population in the intestinal mucosa is comprised of a dynamic continuum ranging from actively proliferating stem cells to terminally differentiated enterocytes lacking mitotic activity. Loss of villus cells must be closely balanced by proliferation in the mitotically active crypt region with the subsequent maturation of these cells into the highly differentiated functional absorptive cells. Mitotic activity is confined to the crypt region and must be finely tuned to the rapid rate of loss of mature enterocytes from the villus. Crypt cell production may be regulated by negative control with suppression of proliferation mediated through a factor produced in the villus. It is unclear whether the rate of subsequent cellular differentiation is intrinsically linked to the rate of proliferation, or whether this process is independently controlled.

Both term and preterm infants have some degree of carbohydrate malabsorption. This may be related to the lactose load in the diet of healthy term infants which may exceed the total capacity of the small intestine to hydrolyze this sugar, or it may be due to the smaller surface area of the neonatal small bowel as compared to that of older infants. Although lactase activity reaches its highest levels at birth, incomplete lactose absorption may be normal in healthy term infants. Indeed, infants who are malabsorbers are more commonly formula or mixed fed than breast fed (19).

The enterohepatic circulation of bile salts is important for the maintenance of a bile salt pool of sufficient size and concentration to permit absorption of dietary lipid. The factors involved in the maintenance of a sufficient bile salt pool include the rate of hepatic bile salt synthesis, the concentration of bile salts by the gallbladder and efficient reabsorption of bile salt conjugates in the intestine. The bile salt pool size in newborn infants is decreased when compared with surface area-matched healthy adults. Experimental observations in immature laboratory animals and in human infants suggest that active transport of bile acids in the ileum is not fully operational and that the bile salt pool is diminished during the fetal and neonatal period. Bile salt transport is mediated by a sodium dependent co-transport system that is similar to that described for glucose, amino acids and inorganic phosphates.

Taurocholate transport into rat ileal brush border membrane vesicles is incompletely developed in two-week-old sucking rats but becomes fully expressed by the time of weaning at three weeks of age (20). Kinetic analysis suggests that maturation of the taurocholate carriers occurs near weaning, primarily through an increase in functional bile acid carriers within the ileal brush border membrane (21). Using brush border membrane vesicles, sodium enhanced uptake of taurocholate appeared abruptly and was first apparent on day 17 and an overshoot uptake was demonstrated on day 18. The lower maximal transport rate and higher apparent Michaelis, constant in young as compared with adult rats, likely reflected a difference in the number of functional bile acid carriers. The change in these kinetic constants could also reflect developmental alterations in the physical character of the membrane itself, with subsequent enhancement of carrier-substrate interaction. The appearance of active ileal transport of bile acids parallels other significant developmental changes such as increasing plasma lev- els of endogenous corticosteroid and thyroxin, alterations in membrane microenvironment and expansion of the bile acid pool (resulting from coexistent maturation of hepatic uptake, synthesis and excretion). It remains unclear what is the importance of glucocorticoids in the development of ileal active bile salt transport and this has been questioned (22).

Thus, while glucocor ticoid treatment promotes precocious development of some intestinal transport systems such as magnesium, calcium and glucose dependent sodium transport and vitamin B12 absorption, this effect is not uniform. Preterm infants whose mothers received dexamethasone or phenobarbital have been found to have larger bile salt pools, increased bile salt synthesis and reduced turnover rates for cholate and chenodeoxycholate compared to similarly aged infants of untreated mothers; an influence of dexamethasone on the intestinal contribution to the maintenance of bile salt pool size appears unlikely.

In both rabbits and rats, there are increases in the cholesterol to phospholipid ratio and a corresponding decrease in membrane fluidity in ileal brush border membrane at the time of weaning (23). With increasing post natal age, the microvillus membrane cholesterol content rises while the phospholipid content remains unchanged. Simultaneously, the microvillus membrane fatty acid composition changes from predominantly saturated to unsaturated species in both jejunum and ileum (23). However, the absence of fluidity change at $37^{\circ} \mathrm{C}$ suggests that the association of alterations in active ileal bile salt transport and microvillus membrane lipid composition and fluidity may not be physiologically significant or interrelated.

What are the factors responsible for the ontogenic expression of intestinal digestive and transport function? Regulatory factors that may initiate maturational changes include the pituitaryadrenal-thyroid secretion. While these hormones may modulate the developmental changes, the initiation of intestinal maturation is not dependent upon these factors. For example, studies using a transplantation technique in which jejunal isographs from newborn rats were 
implanted under the skin of newborn hosts, demonstrated an ontogenic timing mechanism in the jejunum which initiates the expression of sucrase activity (24). This activity is modulated by extrinsic systemic and luminal factors, but the timing for sucrase activity expression appears to be a stringent inherent property of the tissue itself. The cellular basis for the timing of the expression of sucrase activity is unknown but may be mediated by the number of cell divisions. The weaning period is characterized by a shift from a liquid, fat rich, low iron diet supplied by the mother to a solid diet which is rich in carbohydrates and iron. The proportion of carbohydrate relative to fat and the iron content of the weaning diet are critical for the maturation of intestinal microvillus enzymes but not for the ontogenic changes in DNA synthesis.

Initiation of changes in intestinal cell proliferation, DNA synthesis and mucosal enzymes are not diet dependent, but may be triggered by the interaction of the genetic program and hormonal mediators. The protein content of the weaning diet modulates the ontogenic changes in DNA synthesis and appears to be a factor in the completion of the growth of the gastrointestinal tract (25). The exact mechanism by which luminal nutrients modify this developmental process remains an enigma. However, the practical impact of, for example, the use of total parenteral nutrition for nutritional support of infants with disorders of the gastrointestinal tract is clear, with reduced development of stomach, small bowel and pancreatic growth (26). Thus, despite normal weight gain, total serum protein and hematocrit concentrations, intravenously fed animals failed to develop normally.

Dietary insult at an early age may affect the normal development of some of the functional properties of the gastrointestinal tract. These effects may continue into later life and impair later development of the intestinal tract. For example, a two week period of undernutrition in the rat followed by later nutritional rehabilitation is associated with significantly elevated intestinal lactase activity as compared with a well nourished control group (27). The protein content of the weanling diet modulates the ontogenic changes in DNA synthesis and may be a factor in the completion of the growth of the gastrointestinal tract (26). However, while diet plays an important role in maintaining intestinal lactase activity during the sucking period (28), such luminal factors likely have a limited temporal role.

Is intestinal transport of sugars and amino acids subject to critical period programming? The brilliant pioneering work of Karasov and co-workers (29) has raised this important question. Critical period phenomena are events in which a biological mechanism is turned irreversibly on or off only once during an individual's lifetime in response to conditions prevailing at some critical stage. Early diet changes in the carbohydrateprotein ratio have irreversible effects on gut and body size, but early changes in dietary composition of these macronutrients do not appear to exert specific irreversible effects on transport of glucose, choline, leucine, lysine or aspartate. It is unclear why early diet had irreversible anatomic effects which persisted when mice were switched to the same or opposite rations at an early age, but did not have a specific effect on glucose transport independent of the effect on intestinal mass.

Understanding of the regulatory mechanism of intestinal differentiation originates mainly from studies on the rat and mouse. However, the morphological and functional development of the human intestine is unlike that of the rat and mouse: the fetal human intestine organizes villi covered with well differentiated enterocytes during the end of the first term in utero. By 12 weeks of gestation, maltase and sucrase show an activity about half that of a young child. Alkaline phosphatase is present at 10 weeks and rises until week 23 of gestation. Lactase activity remains low during the entire gestational period and increases during the last weeks of pregnancy. Using organ culture of human fetal small intestine, specific increases of lactase and alkaline phosphatase activities were induced by the addition of hydrocor tisone to the culture medium. As well, DNA synthesis was increased, together with an increase in the labelling index of the epithelial cells (30).

Factors controlling enzymatic maturation during ontogenesis of the gut are now being examined in human fetal intestine maintained in organ culture (31). The addition of dexamethasone, insulin or amniotic fluid to the culture medium did not fur ther enhance brush border enzyme activities, except for lactase, the levels of which were doubled by the dexamethasone. Thus, in addition to the differences which exist among mammalian species in the timing of enzyme development, there may be a species specificity in the factors involved in fetal enzymatic maturation. Feeding amino acids with a two or three carbon skeleton (except for cysteine, but including D-amino acids and nonmetabolizable amino acids) strongly induced intestinal ornithine decarboxylase activity. The amino acids that induce hepatic ornithine decarboxylase have no specific structural characteristic. Accumulation of amino acids seems to be necessary for induction of hepatic ornithine decarboxylase because, regardless of the administration route, the enzyme was induced However, humoral factors do not appear to be related to induction of intestinal ornithine decarboxylase, because the enzyme was induced only in the segment perfused with solutions of specific amino acids (32).

The process of ageing alters the structure and function of the small intestine. The epithelial cells that line small intestinal crypts and villi exhibit proliferation, migration and differentiation. During differentiation, enterocytes lose the ability to synthesize DNA and acquire specific enzymes such as the disaccharidases, alkaline phosphatase and enzymes of the DNA salvage pathway. The order pattern of enzyme expression is maintained in ageing rats but the initiation and duration are delayed (33). Because peak specific enzyme activities are similar in young and ageing animals, the reduced specific activities in mucosal homogenates from ageing animals must be due to an increase in the proportion of relatively undifferentiated villus epithelial cells.

From the time of birth to senescence, there are alterations in the digestive and transport function of the intestine. Many 
of the investigations have been performed in rodents and have shown, for example, a reduction in the carrier mediated absorption of valine and lysine (34). This suggests that the activity (number) of carrier molecules for absorption of these amino acids declines with ageing

The effect of ageing on the absorption of some nutrients is likely a specific effect on the uptake mechanism rather than any effect on the morphology of the small bowel (35). For example, the transport of phosphate across the small intestine consists of two components, a saturable electroneutral sodium dependent component and an unsaturable sodium independent component. The energy driving the first component is provided by an extracellular to intracellular sodium gradient maintained by the sodium potassium ATPase at the basolateral membrane, Both components of phosphate uptake decline with ageing (36).

There is increased intestinal permeability to low molecular weight polyethylene glycol in old as compared with young rats (37.38), suggesting that the intestinal protective barrier to the absorption of potentially harmful environmental substances may be less efficient in ageing animals. However, the absorption of some solutes such as xylose are unaffected by ageing (39).

\section{DIABETES MELLITUS}

The gastrointestinal tract is frequently affected in diabetes mellitus. There may be reduced peristalsis and dilatation of the esophagus, gastric retention, disordered small intestinal movement and colonic atony or megacolon. Small bowel transit may be rapid and there may be diminished intestinal tone in some patients with diabetic diarrhea, whereas other patients may have delayed transit through the ileum and increased intestinal motor activity. There may be absent post prandial gastrocolonic responses in diabetic patients suffering from constipation

Enhanced absorption of amino acids, glucose, bile acids, phosphates, cholesterol, fatty acids and fatty alcohols has been reported in rats with experimental diabetes $(40,41)$. Acute diabetes mellitus in the rat is associated with enhanced sodium dependent uptake of phosphate into jejunal brush border membrane vesicles (42). This was likely due to an increase in the activity of sodium-phosphate co-transporters rather than due to any change in the sodium driving force for transport, a gradient maintained by the sodium potassium ATPase in the basolateral membrane.

There are also increased zinc concentrations in the many organs in diabetic animals; this is likely due to a combination of diabetes induced hyperphagia and altered absorption and retention of zinc. Results from an isotope dilution study have indicated that diabetic, but not control, animals are capable of absorbing increased zinc in response to enhanced levels of dietary zinc and that the amount of zincexcreted by the intestine is less in diabetic than control animals (43). Thus, decreased intestinal excretion of endogenous zinc may be a homeostatic response to the increased urinary excretion of endogenous zinc in the diabetic rats and may also lead to elevated zinc concentrations observed in some organs of the diabetic rat.

These alterations in absorption have been associated with changes in the maximal transport capacities, passive permeability properties of the intestine, alterations in unstirred water layers, increased mucosal mass, alterations in brush border membrane lipid content and recruitment of additional brush border carriers for sugar, probably in the mid-villus to crypt region (44).

Diarrhea can be one of the most troublesome gastrointestinal complications in diabetes. Patients with this problem are usually insulin dependent, have autonomic neuropathy and have little or no evidence of nutrient malabsorption or enteropathy. Frequently the diarrhea is intermittent, sometimes alternating with constipation, but may be persistent and severe. Nocturnal fecal incontinence is common and treatment with conventional antidiarrheal agents and antibiotics may be of limited use. Impaired adrenergic regulation of intestinal fluid and electrolyte transport has been suggested as the pathophysiological basis for diabetic diarrhea: these animals have intestinal adrenergic neuropathy, impaired electrolyte absorption and impaired adrenergic innervation. The dia- betic diarrhea occurring in chronic diabetic rats appears to be due to impaired adrenergic regulation of mucosal ion transport accompanied by a post synaptic denervation hypersensitivity that can be reversed by clonidine (45).

In recent years, much effort has been concentrated on reduction of post prandial blood glucose levels in patients with diabetes mellitus by dietary manipulation. Ingestion of viscous polysaccharides with meals may delay carbohydrate absorption by impairing mixing in the small intestine and also by slowing the delivery of food into the small intestine from the stomach. Incorporation into the diet of slowly or incompletely digested carbohydrate in the form of legumes offers another way of retarding glucose absorption. Infusion of fat emulsions into the ileum or duodenum also slows the transit of a meal through the stomach and small intestine of human volunteers and significantly reduces or abolishes the immediate post prandial rises in blood glucose and insulin (46). While ileal perfusion may have limited practical application, feeding a high linoleic acid diet diminishes the enhanced intestinal uptake of glucose and galactose in diabetic rats (47).

Histologic studies of the myenteric plexus of human large and small intestine have revealed no abnormality specific for the diabetic state, although swelling, 'irregularity' and disruption of the parasympathetic fibres within the myenteric plexus of the esophagus have been noted. Electrical field stimulation studies performed in diabetic rats demonstrated lower atropine-sensitive contraction in strips from distal longitudinal muscle in diabetic as compared with nondiabetic control animals (48). This suggests that streptozotocin induced diabetes mellitus is associated with defective cholinergic neuromuscular transmission in the myenteric plexus of the distal small intestine. Insulin therapy seems to improve this abnormality. Insulin receptors have been identified on rat intestinal cells and insulin injection, as well as islet cell transplantation, normalize the enhanced intestinal uptake of hexoses and lipids in diabetic rats (49). While there appears to be no significant changes in gastrointestinal muscarinic receptors 
and adenylate cyclase activity in experimental diabetes, the $B$-adrenergic and serotonergic receptor activities are significantly decreased (50). Diabetic rats have a marked decrease in calcitoningene related peptide-like immunoreactivity nerve fibres around the ganglion cells of the myenteric plexus of ileum and proximal colon, without associated change in substance P-like immunoreactivity in the enteric system (51). There is also effective cholinergic neuromuscular transmission in the myenteric plexus of the distal small intestine of diabetic animals, improved with insulin therapy (48).

Diabetes alters metabolic activities in the enterocyte: acute insulin deficiency (induced by injection of anti-insulin serum) diminishes the rate of lactate production from glucose in jejunal segments in vitro and is accompanied by inhibition of phosphofructokinase and pyruvate dehydrogenase. Lipogenesis and cholesterol synthesis share a common precursor, acetyl $\mathrm{Co} A$, derived from pyruvate generated by intestinal glycolysis. The lower activities of phosphofructokinase and pyruvate dehydrogenase in the intestine would be expected to decrease the availability for lipogenesis of acetyl CoA formed from glucose. However, the rate of lipogenesis in rat small intestine increases on oral glucose loading and decreases after induction of acute insulin deficiency (52). The impairment of lipogenesis can be partially reversed by administration of insulin and parallel changes in the rate of lipogenesis are found in the liver.

Lipogenesis is known to be inhibited by high levels of dietary fat and polyunsaturated fatty acids generally have the greatest effect. Dietary linoleic acid is much more potent than sucrase in reducing hepatic $\triangle 9$ desaturase activity. Both fatty acid synthesis and $\triangle 9$ desaturase are insulin dependent. There is no relationship between $\triangle 9$ desaturase activity and either plasma insulin concentrations or rates of insulin release when assessed in both obese and lean male Zucker rats (53). However, hepatic microsomal $\triangle 9$ desaturase activities are inversely related to the proportion of linoleic acid in liver lipid. Thus, changes in plasma insulin concentration are not primarily respon- sible for diet induced variations in the activity of hepatic microsomal $\triangle 9$ desaturase activity. While $\triangle 9$ desaturase activity of rat liver can be reduced by chemical diabetes and increased by insulin injection, changes in insulin secretion within the physiological range are probably not the primary regulator of this enzyme activity. The potential role of using polyunsaturated fatty acid diets in the treatment of diabetes mellitus needs to be explored in man.

\section{GASTROINTESTINAL PEPTIDES}

The endocrine cells of the digestive system, the amines, peptides and their modes of action have recently been reviewed (54). The distribution of regulatory peptides in human jejunum has been reported (55). Gastrin, secretin, gastric inhibitory polypeptide, enteroglucagon and neurotensin immunoreactivity were almost confined to the endocrine cell-containing mucosal epithelium and only minor amounts of motilin were detected in nonepithelial layers. Vasoactive intestinal polypeptide, substance $\mathrm{P}$ and mammalian bombesin were virtually limited to nonepithelial layers. Only somatostatin was found in all layers. Vasoactive intestinal polypeptide: Vasoactive intestinal polypeptide is found in the central and peripheral autonomic nervous system and is abundantly present in the submucosal neurons that project onto the intestinal mucosa. Vasoactive intestinal polypeptide has the potential of having a putative neurotransmittor role acting locally as a paracrine substance, or at a distant target as a hormone. The vasoactive intestinal polypeptide stimulation of intestinal secretion and inhibition of sodium chloride absorption is associated with a rise in cyclic AMP within enterocytes. Vasoactive intestinal polypeptide also increases excitability of myenteric neurons, enhances muscarinic cholinergic transmission in sympathetic and parasympathetic ganglia and increases the affinity of muscarinic receptors. Vasoactive intestinal polypeptide and other substances that simulate secretion by increasing cyclic AMP levels in enterocytes potentiate the calcium dependent, cholinergic phase of the chloride secretory response evoked by neural stimulation, but do not act as a mediator of the noncholinergic phase of this reponse (56).

Vasoactive intestinal polypeptide inhibits the response of B 10 lymphocytes to concanavalin $\mathrm{A}$ and phytohemagglutinin (57). Duodenal acidification releases vasoactive intestinal polypeptide. as does a meal containing a high fat concentration (58). Vasoactive intestinal polypeptide is also a potent vasodilator that can activate vascular smooth muscle adenylate cyclase and thus potentially contributes to the regulation of vascular tone (59). Vasoactive intestinal polypeptide induces contractions of longitudinal smooth muscle directly as well as indirectly by the stimulation of both cholinergic neurons and noncholinergic excitory neurons (60). As a neurotransmitter, vasoactive intestinal polypeptide is released adjacent to its target tissue and is then rapidly degraded. A vasoactive intestinal polypeptide receptor antagonist has been developed (61).

Motilin: During the interdigestive state in humans and dogs, there is a cycling migrating motor and electrical complex in the gastrointestinal tract and a periodic cycling in the plasma concentration of motilin. Cycling changes in plasma motilin concentration reach a peak during initiation of complexes in the stomach. This observation has led to the suggestion that motilin initiates the migrating motor complex in that site.

Motilin is a gastrointestinal hormone in the dog responsible for induction of the phase III component of the interdigestive migrating motor complex. Serotonin (5-hydroxytryptamine) is present in the enterochromaffin cells. Both 5hydroxytryptophan and hydroxytryptamine initiate a phase III-like activity (62) Motilin appears to initiate phasic contractility in canine jejunum through the cholinergically mediated release of mucosal serotonin (63). Motilin is found in the mucosal cells of the small bowel and in the brain in the pineal gland, pituitary, hypothalamus and the Purkinje cells of the cerebellum. However, initiation of naturally occurring migrating motor complexes in the dog may not be dependent on endogenous release of motilin from the central nervous system (64). 
Corticotropin releasing factor: Corticotropin releasing factor is a hypothalamic hormone which stimulates the secretion of adrenocorticotrophic hormone and $B$-endorphins when injected by the intracerebral ventricular route. Corticotropin releasing factor blocks the cycling peaks of plasma motilin and suppresses migrating motor complexes (65).

Somatostatin: Circulating plasma somatostatin may be a physiological modulator of motilin release and the plasma motilin decline seen during infusion of pharmacological doses of secretin may be explained by the secretin induced somatostatin release which occurs simultaneously (66). The effect of endogenous somatostatin on the gastrointestinal tract has recently been reviewed (67) This hormone is thought to regulate in some way the absorption and secretion processes of the intestine as well as the homeostasis of ingested nutrients. Somatostatin has an inhibiting effect on the secretagogue action of vasoactive intestinal polypeptide mediated by cyclic AMP, increases chloride and sodium absorption in rabbit ileum, inhibits intestinal absorption of glucose and xylose. triglycerides, calcium, amino acids and galactose (68).

Somatostatin suppresses cholera toxin induced secretory diarrhea and the appearance of glycoenzymes in the intestinal lumen and lymph, but does not effect the elevated mucosal cyclic AMP concentration (69). This suggests that the inhibitory facet of somatostatin acts on a process beyond cyclic AMP formation.

Recently, an octapeptide somatostatin analogue (SMS 201-997) has been developed. It is structurally stabilized against enzymatic degradation, has a circulating half-life of approximately 70 mins, can be given by subcutaneous injection and has a dramatically beneficial effect on diarrhea caused by VIPomas or ileal resection (70). Mouth to cecum transit time, as measured by the appearance of nitrogen in the breath, is prolonged by approximately fourfold after administration of this somatostatin analogue (71). It also increases fecal fat excretion, suppresses serum concentrations of galactose after duodenal infusion, depresses duodenal trypsin, lipase and bilirubin increases in response to endogenous stimulation by a Lundh meal, abolishes cholecystokinin release and gallbladder contraction, and diminishes pancreatic amylase, trypsin and lipase output after stimulation with cholecystokinin (72).

Serotonin: Many hormones, including serotonin (5-hydroxytryptamine), substance P, gastrin, motilin, cholecystokinin, somatostatin and vasoactive intestinal polypeptide have been demonstrated within the gastrointestinal tract lumen, either under basal conditions or after vagal stimulation. Endoluminal release of serotonin, substance $\mathrm{P}$ and motilin occurs after electrical vagal stimulation as well as after local increases in mucosal blood flow. Serotonin and substance $\mathrm{P}$ are potent secretagogues on the proximal jejunum and substance $\mathrm{P}$ may act as a local modulator of gastrointestinal blood flow (73). Release of serotonin into the intestinal lumen is increased after vagal nerve stimulation, feeding and increasing intraluminal pressure. This intraluminal release of serotonin plays a physiologic role in the modulation of intestinal motility (74).

Serotinin is a potent intestinal secretagogue in in vitro and in vivo animal models, and intravenous 5-hydroxytryptamine infusions (which produce circulating blood serotonin concentrations similar to those seen in the carcinoid syndrome) produce significant jejunal secretion of water and electrolytes. Because patients with carcinoid syndrome frequently have coexisting hyperserotonemia diarrhea, a causal relationship has been proposed. Other intestinal secretagogues such as substance $\mathrm{P}$ and prostaglandins have also been found in supranormal circulating concentrations in patients with this disorder. Serotonin stimulates net secretion of water and electrolytes without altering mucosal adenylate cyclase activity, cyclic AMP or cyclic guanosine monophosphate (GMP). The calcium dependent electrolyte transport process is present in the intestine and is stimulated by serosal application of 5-hydroxytryptamine, substance $\mathrm{P}$, neurotensin and carbachol. Thus, calcium may be important in modulating the effects of serotonin (75).
Angiotensin: Extracellular volume expansion with isotonic saline leads to an inhibition of ion and water absorption from the jejunal lumen of animals maintained on a normal sodium diet. In contrast, extracellular volume expansion fails to suppress jejunal absorption in animals maintained on a low sodium diet. Presumably saline infusion leads to hemodilution and a reduction in plasma oncotic pressure, which alters the balance of Starling forces across enteric capillaries and thereby favours fluid secretion into the intestine. A hormonal factor is not implicated in the jejunal response to volume expansion and decreased jejunal absorption in animals given a high sodium diet is not due to volume expansion or to inhibition of the renin-angiotensin-aldosterone axis (76). Inhibition of the renin-angiotensin system prevents the elevation of jejunal absorption observed after volume depletion and angiotensin when it must first be converted to angiotensin II before jejunal absorption is stimulated (77).

Intravenous infusion of angiotensin II at doses close to the circulating concentration of the peptide enhances jejunal absorption. This increase in jejunal absorption following volume depletion can be prevented by captopril and by an angiotensin receptor antagonist. Local generation of angiotensin II may play a role in the control of intestinal absorption following extracellular volume depletion. The jejunum is the site of considerable angiotensinase activity and the increase in jejunal absorption following addition of angiotensin 1 can be prevented by inhibition of jejunal converting enzyme (78).

Cholecystokinin: Peptides with cholecystokinin immunoreactivity are known to occur in endocrine cells of the small intestine as well as in central and peripheral neurons. Cholecystokinin-like peptides present in canine brain and intestine have been subjected to microsequence analysis and cholecystokinin-5 is the major one playing a physiological role in brain function (79). L-tryptophan may directly regulate the release of cholecystokinin and membrane depolarization and intracellular generation of cyclic AMP may play a role in activating cho- 
lecystokinin-containing cells (80). The action of cholecystokinin on smooth muscle is either direct or a mixture of direct and neurally mediated effects. The direct effect is usually contractile, whereas the neurally mediated effect may be contractile or inhibitory.

There appears to be a difference in sensitivity of cholecystokinin receptors between muscle cells and neurons from the same location as well as differences in sensitivity between muscle cells from various locations (81). Cholecystokinin may be a 'physiological' mediator of intestinal motility in humans and responses of the intestine to the peptide would appear to vary regionally (82).

Galanin: Galanin is a 29 amino acid peptide which occurs in the brain and intestine of rats, pigs and man. Two molecular forms exist in humans (83) and it is found exclusively in nerves (84). The abundance of galanin in the innervation of the mammalian gut and its reported action on smooth muscle contractility suggests that this peptide is a novel regulatory factor in the control of bowel function.

Substance P: Substance P is contained in enterochromaffin cells as well as within nerves of the intestinal plexuses. Electrical stimulation of the vagus provokes simultaneous intraluminal release of 5-hydroxytryptamine, substance $P$ and motilin. The release of 5-hydroxytryptamine and substance $P$ appears to be under both cholinergic and adrenergic control (85).

Others: Intraluminal gastrin enhances galactose and glycine absorption in the small intestine of rat (86).

Pancreatic polypeptide is localized in the endocrine cells of the pancreas and released after a feed (particularly protein) or cholinergic stimulation. The plasma levels of pancreatic polypeptide cycle with migrating motor complex, peaking in the late phase II in the foregut. It has been suggested that this hormone could be responsible for the transformation of fasted to fed type motility pattern that occurs after a meal. Pancreatic polypeptide does not appear to play any role in fasted intestinal motility, but may contribute to the post prandial suppression of migrating motor complexes (87).
Bombesin is located in the brain, gut and skin of mammals. The inhibitory effect of this peptide on gastrointestinal transit, resulting from intrathecal administration of this tetradecapeptide, depends upon an intact pituitary-adrenal axis (88).

\section{SECRETION, ABSORPTION AND DIARRHEA}

Intestinal ion transport and diarrheal disease have been reviewed (89) and the prospects for new agents in antidiarrheal therapy have also been considered (90). The role of corticosteroid in intestinal ion transport has been reviewed (91).

Cholinergic agonists stimulate small intestinal secretion, whereas adrenergic agonists enhance absorption of salt water. Cholinergic and adrenergic binding receptors have been demonstrated on intestinal epithelial cells. Vagal stimulation induced by sham feeding reduces absorption of salt and water from the human jejunum and psychological stress induced by dichotomous listening reduced water, and sodium chloride absorption, without altering intestinal motility (92). This effect may be mediated by a cholinergic parasympathetic nervous mechanism.

Activation of local nervous reflexes plays an important role in the pathogenesis of several different types of secretion. The intestine is supplied with extrinsic adrenergic fibres of sympathetic origin. Alpha-adrenergic agonists decrease short circuit current by reducing electrogenic anion secretion (bicarbonate and/or chloride secretion) and as well, these $\propto$-adrenergic agonists increase electroneutral sodium chloride uptake. Stimulation of the regional sympathetic fibres increases jejunal food and sodium absorption.

In the cat, the sympathetic nervous outflow to the gut controlling fluid transport is reflexly regulated by input from cardiovascular high and low pressure receptors. In man, reducing the circulating blood volume significantly increases net absorption of fluid and sodium chloride without affecting glucose transport (93). This suggests that the activity in the sympathetic nervous system is initiated from unloading of the cardiopulmonary volume receptors and enhances intestinal absorption of fluid and electrolytes, activating the compensatory mechanisms during hypovolemia due to, for example, hemorrhage.

Prostaglandins, particularly those of the E series, stimulate adenylate cyclase activity in the intestine. The metabolic precursor of the prostanoids, arachidonic acid, also increases mucosal cyclic AMP. Agents such as prostaglandins that increase mucosal cyclic AMP evoke water and electrolyte secretion. A number of hormones desensitize as they stimulate, thereby limiting tissue responses to them and rendering tissues refractory to subsequent additions of the hormone. The nucleotide-independent pathways for secretion may be calcium dependent and may involve protein kinase $\mathrm{C}$.

Protein kinase $\mathrm{C}$ stimulation has been shown to have a crucial role in signal transduction with breakdown of inositol phospholipids and release of diacylglycerol. The diacylglycerol then increases the affinity of protein kinase C for calcium in the presence of acidic phospholipids such as phosphatidyl serine. Phorbol esters directly stimulate protein kinase. Protein kinase Cactivity and phorbol ester binding has been demonstrated in isolated intestinal epithelial cells of the pig (94). The role of protein kinase in human diseases requires further study.

After the creation of an ileostomy, a process of adaptation occurs whereby the loss of water in the ileostomy effluent becomes less than the 1.5 to $2 \mathrm{~L} /$ day entering the colon in the intact subject; volumes of the ileostomy effluent are approximately $0.5 \mathrm{~L} /$ day in the first two weeks after an established ileostomy. In the adapted ileostomate, the low volume and high potassium concentration of fasting effluent suggests that sodium and water absorption are continuously stimulated by chronic salt depletion (95).

Diarrhea may occur following cholecystectomy and bile acid malabsorption has been suspected of playing a role in the pathogenesis of this disorder. However, a study in 25 post cholecystectomy patients with persistent diarrhea demonstrated that, in spite of the presence of marked bile acid malabsorption, the aqueous phase concentrations of the dihydroxy bile acids (chenodeoxycholic 
and deoxycholic acids) did not usually reach the secretory level of $1.5 \mathrm{mM}$. Fecal bile acid composition showed a shift from secondary to primary bile acids and the relatively low aqueous concentrations of the bile acids were the result of low solubility due to an acidic fecal $\mathrm{pH}$. There was an inconsistent therapeutic response to cholestyramine. This indicates that in spite of the presence of increased $24 \mathrm{~h}$ stool bile acids, the associated diarrhea is not necessarily bile acid induced (96). Diarrhea may occur in patients with familial amyloid neuropathy, possibly due to altered gastrointestinal motility due to an autonomic neuropathy. This. in turn, leads to enteric bacterial overgrowth and subsequent diarrhea and steatorrhea which can be temporarily alleviated by cotrimoxazole (97).

\section{BLOOD FLOW}

Evidence for counter current mechanisms in the mammalian gastrointestinal tract has been reviewed (98). Blood flow through the human small intestine and colon has been assessed with laser Doppler flowmetry $(99,100)$. Serial measurements of hydrogen clearance may also be useful in characterizing the role of mucosal blood flow in the pathogenesis of various forms of human disease.

Bile, pancreatic enzymes and undigested food have no effect on intestinal blood flow. Glucose, protein hydrosylates and micellar lipids increase blood flow in the intestine when placed in the lumen. The means by which these nutrients induce a change in vascular resistance is not clear, although changes in tissue metabolism and the release of histamine, vasoactive intestinal peptides, prostaglandins (but not neurotensin) may be involved (101).

Maintenance of intestinal oxygen uptake despite reduction of regional perfusion pressure is necessary to maintain intestinal viability and function. Mature intestine demonstrates preservation of oxygen uptake during hypotension due to a reduction in intestinal vascular resistance; this stabilizes intestinal blood flow and maintains a convective flux of oxygen to the intestinal capillaries. In addition, the diffusive flux of oxygen from capillary to cell increases as perfusion pressure is reduced, as evidenced by an increase in the arteriovenous oxygen content difference across the intestine.

In the neonatal piglet intestine, which is exposed to reduce perfusion pressure. tissue oxygen uptake is maintained by regulation of the arteriovenous oxygen content difference rather than by regulation of blood flow (102). Stimulation of active intestinal transport processes (both absorptive and secretory) are associated with an increase in oxygen uptake. This increase in oxygen uptake is directly related to the rate of transmucosal transport and is met by an increase in blood flow and/or oxygen extraction. Contractions of intestinal smooth muscle may also increase intestinal oxygen uptake and may contribute to the overall oxygen demands of the intestine under conditions of fasting and feeding (103). One of the earliest manifestations of the mucosal damage produced by ischemiareperfusion is enhanced capillary permeability. This results in tissue edema and movement of fluid into the intestinal lumen.

Reactive oxygen metabolites such as superoxide, hydrogen peroxide and hydroxyl free radicals mediate the capillary permeability changes observed in post ischemic mucosal injury. Mucosal protection can be achieved by treatment with the antioxidant enzyme superoxide dismutase or inhibitors of xanthine oxidase. Ischemia and reperfusion may result in xanthine oxidase generated. superoxide dependent accumulation of inflammatory neutrophils in the mucosa where neutrophil derived reactive oxygen metabolites mediate and/or exacerbate intestinal injury (104). Xanthine oxidase is a major source of oxygen free radicals produced during reperfusion of the ischemic small bowel (105). Such therapy is aimed at maintaining cell function by: improving regional blood flow with agents such as vasodilators, positive inotropic agents, membrane stabilizing agents; by providing energy directly to cells by infusion of the high energy phosphate compound ATP; or by administering substrates or compounds that can be utilized within cells under adverse circulatory conditions. This latter approach includes the use of hypotonic glucose in combination with insulin and potassium, which may be useful to reduce the dam- aging effects of ischemia reperfusion on canine small intestine (106).

\section{DRUGS}

Cisapride is a prokinetic agent which facilitates the release of acetylcholine from myenteric nerves. Cisapride thereby enhances spontaneous and electrically increased contractions of intestinal smooth muscle and accelerates the passage of food down the esophagus and out of the stomach. Cisapride induces a continuous phase II-like motor pattern; enhances propulsive activity; induces a prolonged and highly propagative phase II-like jejunal motor activity in fasting humans; and accelerates small bowel transit (107).

Trimebutine is a new therapeutic agent which is used for treatment of irritable bowel syndrome and postoperative ileus. Trimebutine induces a pattern of intestinal motor activity similar to phase III of the digestive motor complex and the motor action of this agent in the small intestine may involve peripheral opiate receptors. Trimebutine induced phase IIIs are accompanied by sharp rises in plasma concentrations of motilin and by simultaneous but opposite effects on gastrin and insulin. It has been suggested that trimebutine influences the generation of activity fronts by altering the equilibrium between stimulatory and inhibitory forces in such a manner that in the post prandial period, the stimulatory mechanism becomes predominant (108).

The potent inhibition of small intestinal motility by $\propto$-adrenoceptor agonists such as clonidine may partially explain their beneficial action in the morphine withdrawal state, as well as in the treatment of some causes of diarrhea (109). The bile salt evoked fluid secretion in the small intestine may result through activation of the enteric nervous system (110).

Vincristine is an effective chemotherapeutic agent widely used in the treatment of various neoplastic disorders. Gastrointestinal symptoms are common and the associated abdominal pain and constipation may be due to histologic damage to the myenteric plexus, as well as alterations of myoelectric activity and associated changes in intestinal transit (111). 


\section{REFERENCES}

1. Bikle DD, Gee EA, Munson SJ. Effect of ethanol on intestinal calcium transport in chicks. Gastroenterology 1986;91:870-6

2. Said HM, Strum WB. Effect of ethanol and other aliphatic alcohols on the intestinal transport of folates. Digestion 1986;35:129-35.

3. Mazzanti R. Debnam ES, Jenkins WJ. Effect of chronic ethanol intake on lactase activity and active galactose absorption in rat small intestine. Gut 1987:28:56-60.

4. Baraona E. Julkunen R. Tannenbaum L, Lieber CS. Role of intestinal bacterial overgrowth in ethanol production and metabolism in rats Gastroenterology 1986;90:103-10.

5. Conteas CN, Demorrow J. Small intestinal amino acid and glucose malabsorption by ethanol-induced sodium channel activation. Biochem Arch 1987:3:85-93

6. Keelan M, Walker K, Rajotte RV Clandinin MT. Thomson ABR. Effect of chronic ethanol and food deprivation on intestinal villus morphology and brush border membrane content of lipid and marker enzymes. Can J Physiol Pharmacol 1985;63:1312-20.

7. Hajiar JJ, Baker ER, Renison DM, Gardner PW, Zirin R, Tomicic TK. Effectof ethanol on choline transport in rat jejunum. Am J Physiol 1985:249:G177-83

8. Zucoloto S, Muccilo G, Wright NA, Alison MR. Chronic effects of alcohol on the epithelium of the small intestine using two experimental models. Virchows Arch 1985;49:365-73

9. Simonowski UA, Seitz HK, Baier B. Kommerell B, Schmidt-Gayk H, Wright NA. Chronic ethanol consumption selectively stimulates rectal cell proliferation in the rat. Gut 1986:27:278-82

10. Beck IT, Morris GP, Buell MG. Ethanol-induced vascular permeability changes in the jejunal mucosa of the dog. Gastroenterology 1986;90:1137-45.

11. Yeh K-Y, Holt PR. Ontogenic timing mechanism initiates the expression of rat intestinal sucrase activity Gastroenterology 1986;90;520-6.

12. Thomson ABR, Keelan M. The development of the small intestine. Can J Physiol Pharmacol 1986;64:13-29

13. Pang KY, Bresson JL, Walker WA Development of gastrointestinal surface VIII. Lectin identification of carbohydrate differences. Am J Physiol 1987:252:G685-91

14. Heyman M, Crain-Denoyelle AM, Corthier G, Morgat JL, Desjeux IF. Postnatal development of protein absorption in conventional and germ-free mice. Am J Physiol 1986;251:G326.

15. Oren AJM, Diver-Haber A, Kaplan B, Passwell J. Effects of the components of breast milk on mucosal enzyme activity of the newborn small intestine. Pediatr Res 1987:21:126-30.

16. Gallo-Payet N, Pothier P. Hugon JS Ontogeny of EGF receptors during postnatal development of mouse small intestine. J Pediatr Gastroenterol Nutr 1987:6:114-20

17. Berseth $\mathrm{Cl}$. Breast-milk-enhanced intestinal and somatic growth in neonatal rats. Biol Neonate 1987;51:53-9

18. Kurokoaw M, Lynch K. Podolsky DK. Effects of growth factors on an intestinal epithelial cell line: Transforming growth factor $B$ inhibits proliferation and stimulates differentiation. Biochem Biophys Res Commun 1987:142:775-82.

19. Roggero P, Mosca F, Motta G, et al. Sugar absorption in healthy preterm and full-term infants. J Pediatr Gastroenterol Nutr 1986;5:214-19.

20. Barnard JA, Gishan FK, Wilson FA. Ontogenesis of taurocholate transport by rat ileal brush border membrane vesicles. J Clin Invest 1985;75:869-73.

21. Moyer MS, Heubi JE, Goodrich AL, Balistreri WF, Suchy FJ. Ontogeny of bile acid transport in brush border membrane vesicles from rat ileum. Gastroenterology 1986;90:1188-96.

22. Heubi JE, Gunn TD, The role of glucocorticoids in the postnatal development of ileal active bile salt transport. Pediatr Res 1985; 19:1147-51.

23. Heubi JE, Fellows JL. Postnatal development of intestinal bile salt transport. Relationship to membrane physicochemical changes. J Lipid Res 1985;26:797-805.

24. Yeh K-Y, Holt PR. Ontogenic timing mechanism initiates the expression of rat intestinal sucrase activity. Gastroenterology 1986;90:520-6

25. Buts JP, Nyakabasa M.Role of dietary protein adaptation at weaning in the development of the rat gastrointestinal tract. Pediatr Res 1985; 19:857-62

26. Goldstein RM, Hebiguchi T, Luk GD, et al. The effects of total parenteral nutrition on gastrointestinal growth and development. J Pediatr Surg 1985;20:785-91

27. Majumdar APN. Effects of undernutrition and subsequent nutritional rehabilitation of hydrocortisone administration on growth and function of the gastrointestinal tract in rats. Nutr Rep Int 1986;33:187-98.

28. Yeh K-Y, Holt PR. Rat milk maintains intestinal lactase activity in rat pups whereas artificial formulas do not. Pediatr Res 1985:19:963-7.
29. Karasov WH, Solberg DH, Chang SD Hughes M, Stein ED, Diamond JM. Is intestinal transport of sugars and amino acids subject to critical-period programming? Am J Physiol 1985:249:G770-85.

30. Arsenault P. Menard D. Influence of hydrocortisone on human fetal small intestine in organ culture. J Pediatr Gastroenterol Nutr 1985;4:893-901.

31. Simon-Assmann P, Lacroix B. Kedinger M. Haffen K. Maturation of brush border hydrolases in human fetal intestine maintained in organ cultures. Early Hum Dev 1986;13:65-74

32. Minami H, Miyamoto K-I, Fujii Y, Nakabou Y, Hagihira H. Induction of intestinal or nithine decarboxylase by single amino acid feeding. J Biochem 1985:98:133-9.

33. Holt PR, Tierney AR, Kotler DP. Delayed enzyme expression: A defect of aging rat gut. Gastroenterology 1985;89:1026-34

34. Younoszai MK, Smith C, Finch MH Comparison of in vitro jejunal uptake of L-valine and L-lysine in the rat during maturation. J Pediatr Gastroenterol Nutr 1985:4:992-7

35. Corazza GR, Frazzoni M, Gatto MRA, Gasbarrini G. Ageing and small bowel mucosa: A morphometric study. Gerontology 1986:32:60-5

36. Borowitz SM. Ghishan FK. Maturation of jejunal phosphate transport rat brush border membrane vesicles. Pediatr Res 1985; 19:1308-12.

37. Katz D, Hollander D. Said HM Dadufalza V. Ageing-associated increase in intestinal permeability to polyethylene glycol 900. Dig Dis Sci 1987:32:285-8

38. Hollander D. Tarnawski H. Ageing-associated increase in intestinal absorption of macromolecules. Gerontology 1985;31:133-7.

39. Johnson SL. Mayersohn M, Conrad KA. Gastrointestinal absorption as a function of age: Xylose absorption in healthy adults. Clin Pharmacol Ther 1985:38:331-5.

40. Thomson ABR. Diets alter jejunal morphology and brush border membrane composition in streptozotocin-diabetic rats: Can J Physiol Pharmacol 1987;65:210-8

41. Thomson ABR. Dietary fat selectively alters transport properties of rat jejunum. J Clin Invest 1986:77:279-88

42. Ghishan FK, Borowitz S, Mulberg A Phosphate transport by jejunal brush border membrane vesicles of the streptozotocin-diabetic rats. Diabetes 1985;34:723-7.

43. Johnson WT. Canfield WK. Intestinal absorption and excretion of zinc in streptozotocin-diabetic rats as affected by dietary zinc and protein. J Nutr 
$1985 ; 15: 1217-27$

44. Fedorak RN, Chang EB, Madara JL, Field M. Altered Na-dependent nutrient absorption in streptozotocintreated chronically diabetic rats. J Clin Invest 1987:79:1571-8

45. Chang EB, Fedorak RN, Field M. Experimental diabetic diarrhea in rats. Intestinal mucosal denervation hypersensitivity and treatment with clonidine. Gastroenterology 1986:91:564-9.

46. Welch I McL, Bruce C, Hill SE, Read NW. Duodenal and ileal lipid suppresses postprandial blood glucose and insulin responses in man: Possible implications for the dietary management of diabetes mellitus. Clin Sci 1987:72:209-16.

47. Thomson ABR, Keelan M, Clandinin MT, Walker K. A high linoleic acid diet diminishes enhanced intestinal uptake of sugars in diabetic rats. Am J Physiol 1987;252:G262-71

48. Nowak TV, Harrington B, Kalbfleisch HJ. Amatruda HM. Evidence for abnormal cholinergic neuromuscular transmission in diabetic rat small intestine. Gastroenterology 1986:91:124-32

49. Thomson ABR, Rajotte RV. Insulin and islet cell transplantation in streptozotocin-diabetic rats: Effect on intestinal uptake of hexoses. Comp Biochem Physiol (A) 1985:82:827-31.

50. Altan VM, Yildizoglu N, Ozturk Y. Decreased gastro-intestinal responses to certain agonists in streptozotocinand alloxan-diabetic rats in vitro. Pharmacology 1987; 34:143-8.

51. Belai A, Burnstock G. Selective damage of intrinsic calcitonin gene-related peptide-like immunoreactive enteric nerve fibers in streptozotocin-induced diabetic rats. Gastroenterology 1987;92:730-4.

52. Williamson DH, Ilic V. Hughes J. Effects of short-term insulin deficiency on lipogenesis and cholesterol synthesis in rat small intestine and liver in vivo. Biochem J 1985:231:221-3.

53. Weekes TEC, Wahle KW], Lebaijuri MB. Effects of dietary triolein and sunflower oil on insulin release and lipid metabolism in Zucker rats. Lipids 1986;21:220-5.

54. Grube D. The endocrine cells of the digestive system: Amines, peptides, and modes of action. Anat Embryol 1986:175:151-62.

55. Ferri G-L, Adrian TE, Soimero L, et al. Regulatory peptide distribution in separated layers of the human jejunum. Digestion 1987:37:15-21.

56. Cooke HJ. Zafirova M. Carey HV, Walsh JH, Grider J. Vasoactive intestinal polypeptide actions on the guinea pig intestinal mucosa during neural stimulation. Gastroenterology 1987;92:361-70.

57. Kreo C], Gores A, Go VLW. Gastrointestinal regulatory peptides modulate in vitro immune reactions of mouse lymphoid cells. Clin Immunol Immunopathol 1986;39:308-18.

58. Hill P. Thijssen HH, Garbaczewski L. Koppeschaar HPR, Dewaard F. VIP and prolactin release in response to meals. Scand J Gastroenterol $1986 ; 21: 958-60$

59. Ganz P, Sandrock AW, Landis SC. Leopold J, Gimbrone MA Jr. Alexander RW. Vasoactive intestinal peptide: Vasodilatation and cyclic AMP generation. Am J Physiol 1986;250:H755-60

60. Kusunoki M. Tsai LH, Taniyama K, Tanaka C. Vasoactive intestinal polypeptide provokes acetylcholine release from the myenteric plexus. Am J Physiol 1986;251:G51-5.

61. Pandol SJ, Dharmsathaphorn D. Schoeffield MS, Vale W, Rivier J. Vasoactive intestinal peptide receptor antagonist (4C1-D-Phe ${ }^{6}$. Leu ${ }^{17}$ ) VIP Am J Physiol 1986;250:G553-7

62. Schemann M, Ehrlein H-j. 5-Hydroxytryptophan and cisapride stimulate propulsive jejunal motility and transit of chyme in dogs. Digestion 1986:34:229-35

63. Kellum JM, Maxwell RJ, Potter J, Kummerle JF. Motilin's induction of phasic contractility in canine jejunum is mediated by the luminal release of serotonin. Surgery 1986;100:445-53.

64. Hashmonai M, Go VL, Yaksh T, Szurszewski JH. Effect of central administration of motilin on migrating complexes in the dog. Am J Physiol 1987:252:G195-9.

65. Bueno L, Fargeas MJ, Gue M, Peeters TL. Bormans V, Fioramonti J. Effects of corticotropin-releasing factor on plasma motilin and somatostatin levels and gastrointestinal motility in dogs. Gastroenterology 1986;1:884-9.

66. Jenssen TG, Haukland $\mathrm{HH}$. Florholmen J, Jorde R, Burhol PG. Evidence of somatostatin as a humoral modulator of motilin release in man. Scand J Gastroenterol 1986,21:273-80.

67. Lucey MR. Endogenous somatostatin and the gut (progress report). Gut $1986 ; 27: 457-67$

68. Sorribas V. Arruebo P. Murillo D. Alcalde Al. Effect of somatostatin on D-galacrose transport across the small intestine of rats. Comp Biochem Physiol (A) 1987;86:63-6.

69. Yoshioka M. Asakur H. Hamada Y, et al. Inhibitory effect of somatostatin on cholera toxin-induced diarrhea and glycoenzyme secretion in rat intestine. Digestion 1987:36:141-7.

70. Cooper JC. Williams NS, King RFG],
Barker MCJ. Effects of a long-acting somatostatin analogue in patients with severe ileostomy diarrhea. Br J Surg 1986;73:128-31.

71. Fuessl HS, Carolan G, Williams G, Bloom SR. Effect of a long-acting somatostatin analogue (SMS 201-995) on postprandial gastric emptying of $99 \mathrm{~m}$ Te-tin coloid and mouth-tocaecum transit time in man. Digestion 1987:36:101-7.

72. Lembcke B. Creutzfeldt W. Schleser S, Ebert R, Shaw C. Koop I. Effect of the somatostatin analogue sandostatin (SMS 201-995) on gastrointestinal. pancreatic and biliary function and hormone release in normal men. Digestion 1987;36:108-24

73. McFadden D, Jaffe BM, Zinner MI. Effect of luminally administered serotonin and substance $P$ on jejunal handling of water and electrolytes. Am J Surg 1986;151:81-6.

74. Martensson HG, Zinner MJ, Jaffe B Effects of intraluminal perfusion with serotonin on jejunal motility. Dig Dis Sci 1986;31:811-6.

75. Zinner MJ, McFadden D, Sherlock D, Jaffe BM. Verapamil reversal of serotonin-induced jejunal secretion of water and electrolytes in awake dogs. Gastroenterology 1986;90:515-9.

76. Levens NR. Response of rat jejunum to changes in sodium and volume balance, Am J Physiol 1986:251:G413-20.

77. Levens NR, Response of isolated rat jejunum to angiotensin peptides. Am ] Physiol 1986:251:G559-66.

78. Levens NR. Local control of jejunal absorption by the renin-angiotensin system. J Cardiovasc Pharmacol 1986:8(Suppl):S17-22.

79. Shively J, Reeve JR Jr, Eysselein VE, Ben-Avram C. Vigna SR, Walsh JH. CCK-5: Sequence analysis of a small cholecystokinin from canine brain and intestine. Am I Physiol 1987:252:G272-5.

80. Barber DL. Walsh JH, Soll AH. Release and characterization of cholecystokinin from isolated canine jejunal cells. Gastroenterology 1986;91:627-36.

81. Grider JR. Makhlouf GM. Regional and cellular heterogeneity of cholecystokinin receptors mediating muscle contraction in the gut. Gastroenterology 1987:92:175-80.

82. Kellow IE, Miller LJ, Phillips SF, Haddad AC. Zinsmeister AR, Charboneau JW. Sensitivities of human jejunum, ileum, proximal colon, and gallbladder to cholecystokinin octapeptide. Am J Physiol 1987;252:G345-56

83. Bauer FE, Adrian TE, Christofides ND, et al. Distribution and molecular heterogeneity of galanin in human. 
pig. guinea pig, and rat gastrointestinal tracts. Gastroenterology 1986,91:877-85

84. Bishop AE. Polak JM. Bauer FE. Christofides ND, Carlei F, Bloom SR Occurrence and distribution of a newly discovered peptide, galanin, in the mammalian enteric nervous system. Gut 1986:27:849-57.

85. Ferrara A, Zinner MJ, Jaffe BM. Intraluminal release of serotonin. substance $\mathrm{P}$, and gastrin in the canine small intestine, Dig Dis Sci 1987,32:289-94

86. Schwartz MZ, Storozuk RB. Enhancement of small intestine absorption by intraluminal gastrin. J Surg Res 1986:40:421-5

87. Thor PI. Konturek IW, Konturek SI Pancreatic polypeptide and intestinal motility in dogs. Dig Dis Sci $1987.32 .513-9$

88. Kuslo RJ. Gmorek DE. Cowan A. Porreca F. Intrathecal bombesininduced inhibition of gastrointestinal transit: Requirement for an intact pituitary-adrenal axis. Regul Pept 1986:14:237-42.

89. Fondacaro JD. Intestinal ion transport and diarrheal discase. Am J Physiol 1986:250:G1-8.

90. Fedorak RN, Field M. Antidiarrheal therapy. Prospects for new agents Dig Dis Sci 1987:32:195-201.

91. Sandle Gl, Binder HI. Corticosteroids and intestinal ion transport. Gastratenterology 1987:93:188-96.

92. Barclay GR, Turnberg LA. Effect of psychological stress on salt and water transport in the human jejunum. Gastroenterology 1987:93:91-7.

93. Sjovall $\mathrm{H}$. Abrahamsson $\mathrm{H}$, Westlander G, et al. Intestinal fluid and electrolyte transport in man during reduced circulating blood volume. Gut $1986 ; 27: 913-8$
94. Weikel CS. Sando II. Guerrant RL. Stimulation of porcine jejunal ion secretion in vivo by protein kinase- $\mathrm{C}$ activators. I Clin Invest 1985:76:2430-5

95. Ladas SD, Tisaacs PE, Murphy GM. Sladen GE. Fasting and postprandial ileal function in adapted ileostomates and normal subjects. Gut 1986:27:906-12

96. Fromm H, Tunuguntla AK, Malavolti M. Sherman C. Cervak S. Absence of significant role of bile acids in diarrhea of a heterogeneous group of post cholecystectomy patients. Dig Dis Sci 1987:32:33-44

97. Feurle GE. Pathophysiology of diarrhea in patients with familial amyloid neuropathy. Digestion 1987:36:13-7.

98. Jodal M, Lundgren O. Countercurrent mechanisms in the mammalian gastrointestinal tract. Gastroenterology 1986:91:225-41

99. Ahn H, Lindhagen J, Lundgren $\mathrm{O}$. Measurement of colonic blood flow with laser Doppler flowmetry. Scand I Gastroenterol 1986;21:871-80.

100. Ahn H, Lindhagen J, Nilsson GE, Oberg PA, Lundgren O. Assessment of blood flow in the small intestine with laser Doppler flowmetry. Scand 1 Gastroenterol 1986:21:863-70.

101. Gallavan RH Jr, Shaw C, Murphy RF, Buchanan KD, Joffe SN, Jacobson ED Effects of micellar oleic acid on canine jejunal blood flow and neurotensin release. Am I Physiol 1986:251:G649-55

102. Nowicki PT, Hansen NB, Menke JA. Intestinal blood flow and oxygen uptake in the neonatal piglet during reduced perfusion pressure. Am J Physiol 1987;251:G190-4

103. Kvietys PR, Barrowman JA, Harper
SL. Granger DN. Relations among canine intestinal motility, blood flow, and oxygenation. Am J Physiol 1986:251:G25-33

104. Grisham MB, Hernandez LA, Granger DN. Xanthine oxidase and neutrophil infiltration in intestinal ischemia. Am ] Physiol 1986:251:G567-74

105. Granger DN, McCord JM, Parks DA. Hollwarth ME. Xanthine oxidase inhibitors attenuate ischemia-induced vascular permeability changes in the cat intestine. Gastroenterology 1986;90:80-4

I06. Lang CH. Alteveer RJ. Effects of glucose-insulin-potassium on intestinal hemodynamics and substrate utilization during endotoxemia. Am J Physiol 1986:251:G341-8

107. Stacher G, Steinringer $\mathrm{H}$, Schneider $\mathrm{C}$ Winklehner S, Mittelbach G. Gaupmann G. Effects of cisapride on jejunal motor activity in fasting healthy humans. Gastroenterology 1986;90:1210-6

108. Poitras P. Honde C, Havrankova J, et al. Effect of trimebutine on intestinal motility and plasma motilin in the dog Am J Physiol 1986;251: G349-53.

109. Thollander M, Eriksson U. Hellstrom PM, Al-Saffar A, Svensson TH Alpha, ${ }_{2}$-adrenoceptor agonists inhibit the migrating myoelectric complexes of the small intestine in rats. Acta Physiol Scand 1987:130:165-7.

110. Tantisira MH, Jodal M, Lundgren O. Intraluminal bile salt increases rate of firing in afferent fibers from the small intestine of the rat. Experientia 1987:43:168-9

111. Sninsky CA. Vincristine alters myoelectric activity and transit of the small intestine in rats. Gastroenterology 1987:92:472-8.

\section{CORRECTION}

Peutz-Jeghers syndrome without polyposis. Anthony G. Catto-Smith, Mark K. Patrick, D. Grant Gall

Figure 1) Barium contrast study of the upper gastrointestinal tract and small intestine showing no evidence of polyposis

This figure (Can J Gastroenterol 1988;2(3): 118) was unfortunately published inverted. The error is regretted

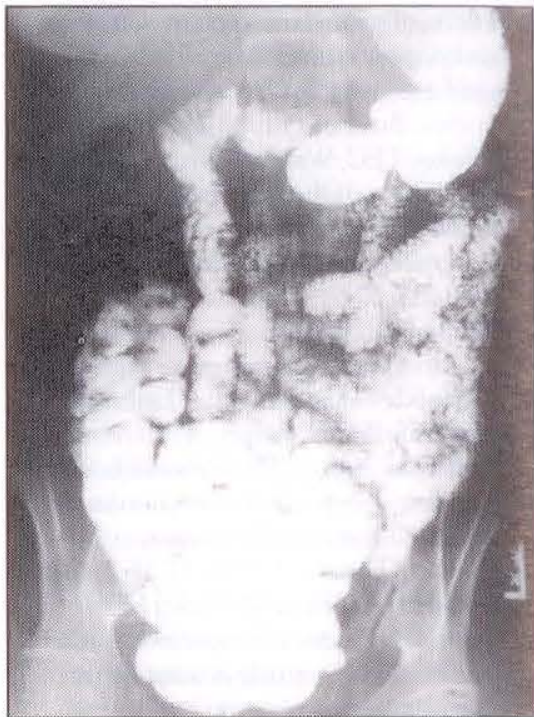




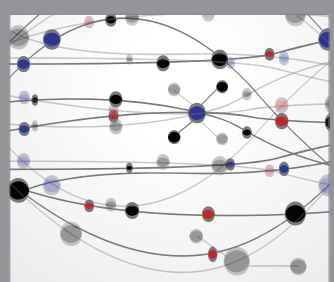

The Scientific World Journal
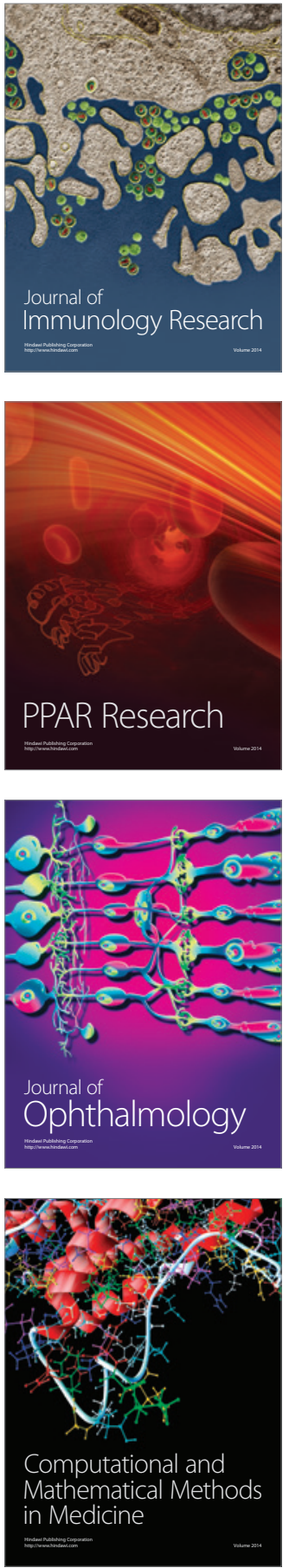

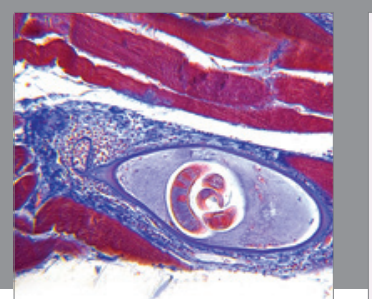

Gastroenterology Research and Practice

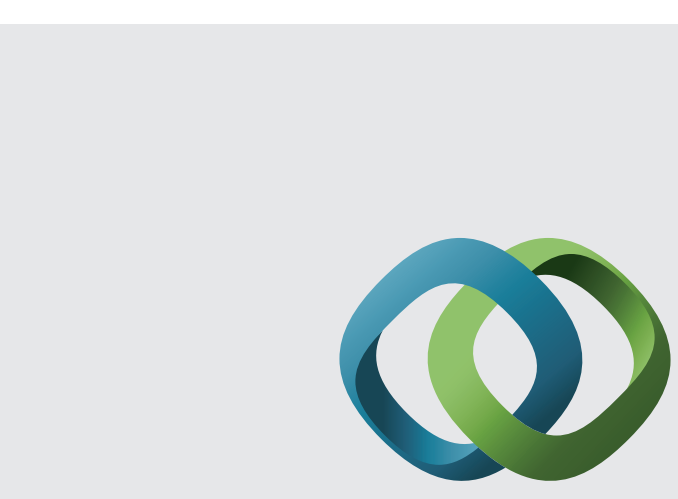

\section{Hindawi}

Submit your manuscripts at

http://www.hindawi.com
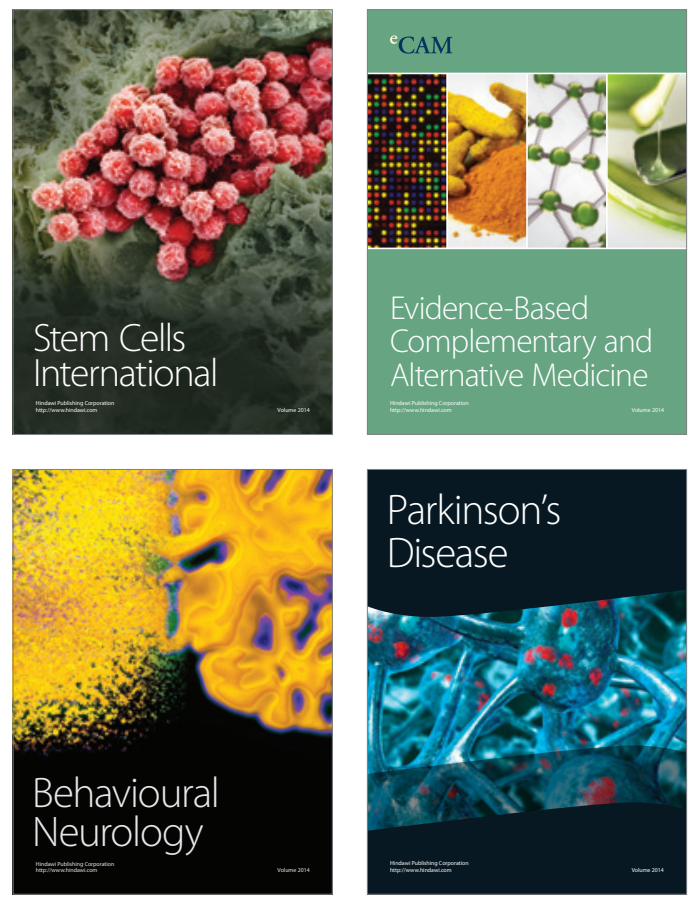
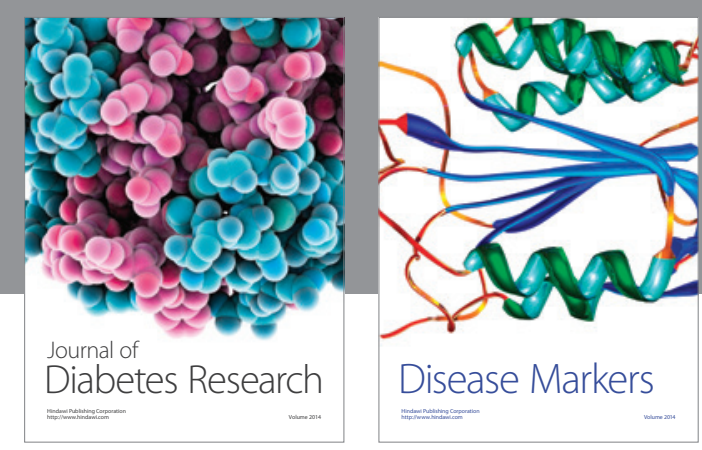

Disease Markers
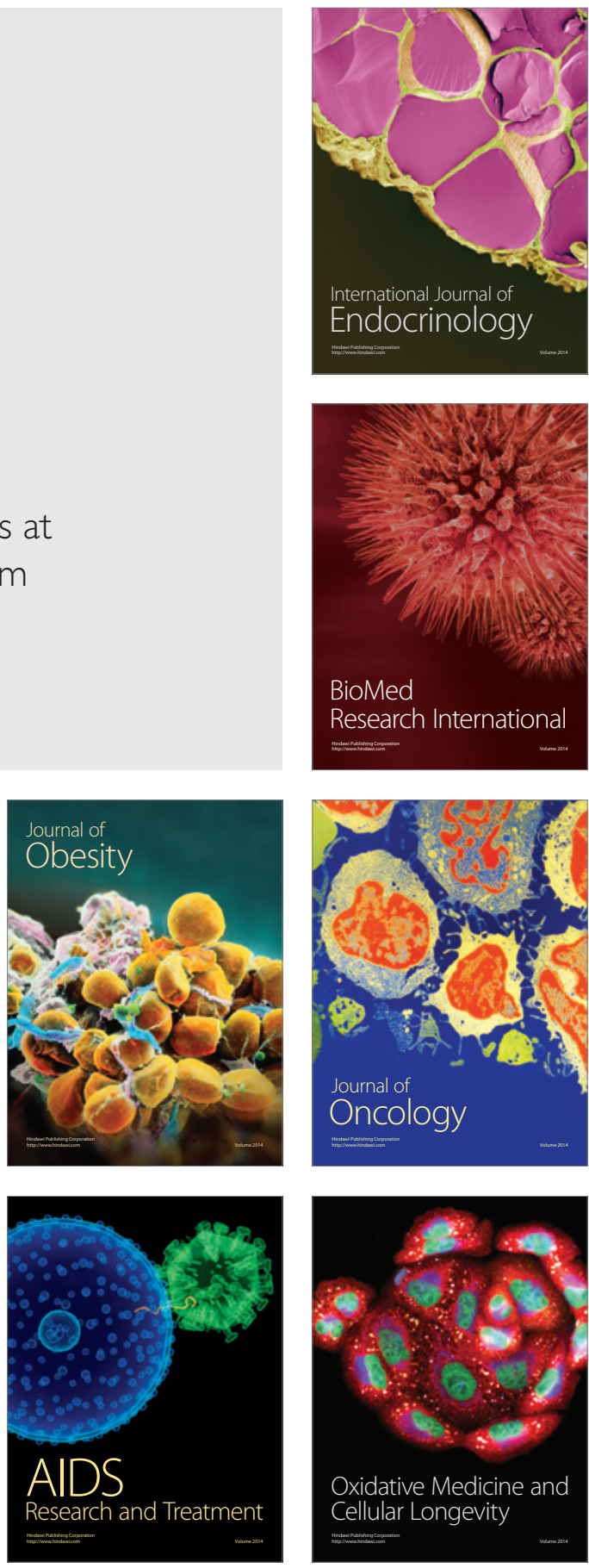